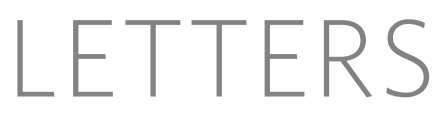

\title{
Genome sequence and analysis of the Irish potato famine pathogen Phytophthora infestans
}

\author{
Brian J. Haas ${ }^{1 *}$, Sophien Kamoun ${ }^{2,3 *}$, Michael C. Zody ${ }^{1,4}$, Rays H. Y. Jiang ${ }^{1,5}$, Robert E. Handsaker ${ }^{1}$, Liliana M. Cano ${ }^{2}$, \\ Manfred Grabherr ${ }^{1}$, Chinnappa D. Kodira ${ }^{1} \dagger$, Sylvain Raffaele ${ }^{2}$, Trudy Torto-Alalibo ${ }^{3} \dagger$, Tolga O. Bozkurt ${ }^{2}$, \\ Audrey M. V. Ah-Fong ${ }^{6}$, Lucia Alvarado ${ }^{1}$, Vicky L. Anderson ${ }^{7}$, Miles R. Armstrong ${ }^{8}$, Anna Avrova ${ }^{8}$, Laura Baxter ${ }^{9}$,
} Jim Beynon ${ }^{9}$, Petra C. Boevink ${ }^{8}$, Stephanie R. Bollmann ${ }^{10}$, Jorunn I. B. Bos ${ }^{3}$, Vincent Bulone ${ }^{11}$, Guohong Cai ${ }^{12}$, Cahid Cakir ${ }^{3}$, James C. Carrington ${ }^{13}$, Megan Chawner ${ }^{14}$, Lucio Conti ${ }^{15}$, Stefano Costanzo ${ }^{16}$, Richard Ewan ${ }^{15}$, Noah Fahlgren ${ }^{13}$, Michael A. Fischbach ${ }^{17}$, Johanna Fugelstad ${ }^{11}$, Eleanor M. Gilroy ${ }^{8}$, Sante Gnerre ${ }^{1}$, Pamela J. Green ${ }^{18}$, Laura J. Grenville-Briggs ${ }^{7}$, John Griffith ${ }^{14}$, Niklaus J. Grünwald ${ }^{10}$, Karolyn Horn ${ }^{14}$, Neil R. Horner ${ }^{7}$, Chia-Hui Hu ${ }^{19}$, Edgar Huitema ${ }^{3}$, Dong-Hoon Jeong ${ }^{18}$, Alexandra M. E. Jones ${ }^{2}$, Jonathan D. G. Jones ${ }^{2}$, Richard W. Jones ${ }^{20}$, Elinor K. Karlsson ${ }^{1}$, Sridhara G. Kunjeti ${ }^{21}$, Kurt Lamour ${ }^{22}$, Zhenyu Liu ${ }^{3}$, LiJun Ma ${ }^{1}$, Daniel MacLean ${ }^{2}$, Marcus C. Chibucos ${ }^{23}$, Hayes McDonald ${ }^{24}$, Jessica McWalters ${ }^{14}$, Harold J. G. Meijer ${ }^{5}$, William Morgan ${ }^{25}$, Paul F. Morris ${ }^{26}$, Carol A. Munro ${ }^{27}$, Keith O’Neill ${ }^{1} \dagger$, Manuel Ospina-Giraldo ${ }^{14}$, Andrés Pinzón ${ }^{28}$, Leighton Pritchard ${ }^{8}$, Bernard Ramsahoye ${ }^{29}$, Qinghu Ren ${ }^{30}$, Silvia Restrepo ${ }^{28}$, Sourav Roy ${ }^{6}$, Ari Sadanandom ${ }^{15}$, Alon Savidor ${ }^{31}$, Sebastian Schornack ${ }^{2}$, David C. Schwartz ${ }^{32}$, Ulrike D. Schumann ${ }^{7}$, Ben Schwessinger ${ }^{2}$, Lauren Seyer ${ }^{14}$, Ted Sharpe ${ }^{1}$, Cristina Silvar ${ }^{2}$, Jing Song ${ }^{3}$, David J. Studholme ${ }^{2}$, Sean Sykes ${ }^{1}$, Marco Thines ${ }^{2,33}$, Peter J. I. van de Vondervoort ${ }^{5}$, Vipaporn Phuntumart ${ }^{26}$, Stephan Wawra ${ }^{7}$, Rob Weide ${ }^{5}$, Joe Win ${ }^{2}$, Carolyn Young ${ }^{3}$, Shiguo Zhou ${ }^{32}$, William Fry ${ }^{12}$, Blake C. Meyers ${ }^{18}$, Pieter van West ${ }^{7}$, Jean Ristaino ${ }^{19}$, Francine Govers ${ }^{5}$, Paul R. J. Birch ${ }^{34}$, Stephen C. Whisson ${ }^{8}$, Howard S. Judelson ${ }^{6} \&$ Chad Nusbaum ${ }^{1}$

\begin{abstract}
Phytophthora infestans is the most destructive pathogen of potato and a model organism for the oomycetes, a distinct lineage of fungus-like eukaryotes that are related to organisms such as brown algae and diatoms. As the agent of the Irish potato famine in the mid-nineteenth century, $P$. infestans has had a tremendous effect on human history, resulting in famine and population displacement ${ }^{1}$. To this day, it affects world agriculture by causing the most destructive disease of potato, the fourth largest food crop and a critical alternative to the major cereal crops for feeding the world's population $^{1}$. Current annual worldwide potato crop losses due to late blight are conservatively estimated at $\$ 6.7$ billion $^{2}$. Management of this devastating pathogen is challenged by its remarkable speed of adaptation to control strategies such as genetically resistant cultivars $^{3,4}$. Here we report the sequence of the $P$. infestans genome,
\end{abstract}

which at $\sim 240$ megabases $(\mathrm{Mb})$ is by far the largest and most complex genome sequenced so far in the chromalveolates. Its expansion results from a proliferation of repetitive DNA accounting for $\sim 74 \%$ of the genome. Comparison with two other Phytophthora genomes showed rapid turnover and extensive expansion of specific families of secreted disease effector proteins, including many genes that are induced during infection or are predicted to have activities that alter host physiology. These fast-evolving effector genes are localized to highly dynamic and expanded regions of the $P$. infestans genome. This probably plays a crucial part in the rapid adaptability of the pathogen to host plants and underpins its evolutionary potential.

The size of the $P$. infestans genome is estimated by optical map and other methods at $240 \mathrm{Mb}$ (Supplementary Information). It is severalfold larger than those of the related Phytophthora species P. sojae

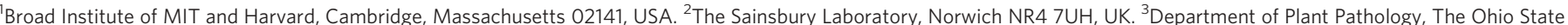

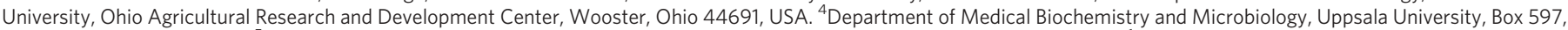

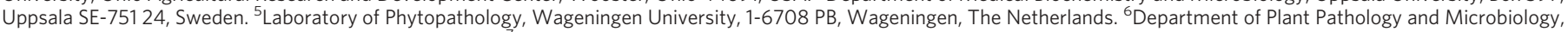
University of California, Riverside, California 92521, USA. ${ }^{7}$ University of Aberdeen, Aberdeen Oomycete Laboratory, College of Life Sciences and Medicine, Institute of Medical Sciences, Foresterhill, Aberdeen AB25 2ZD, UK. ${ }^{8}$ Plant Pathology Programme, Scottish Crop Research Institute, Invergowrie, Dundee DD2 5DA, UK. ${ }^{9}$ University of Warwick,

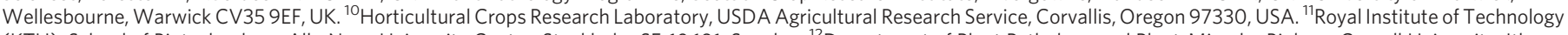

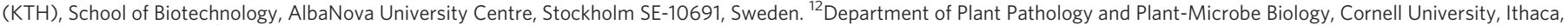

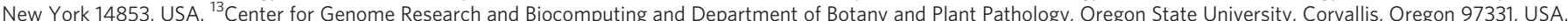

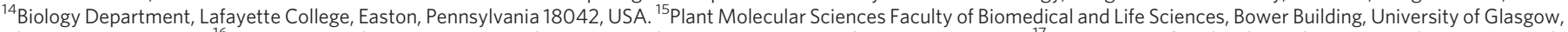

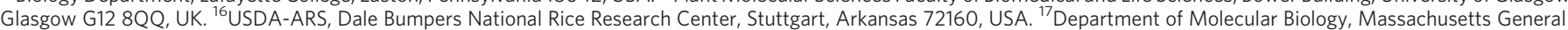

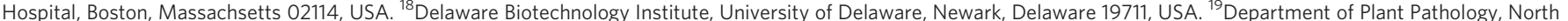

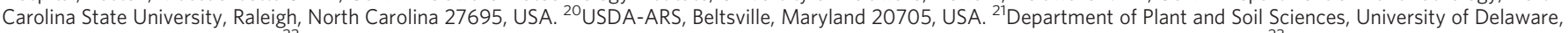

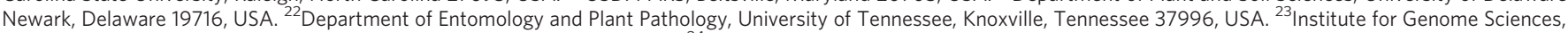

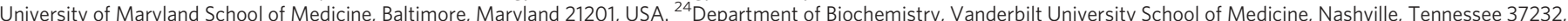

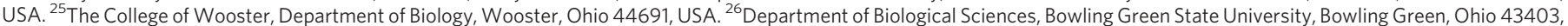

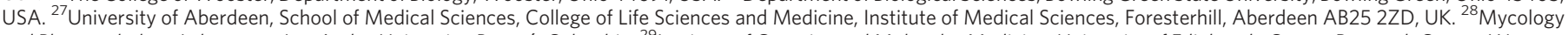

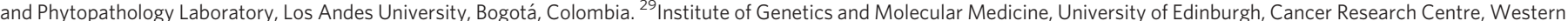

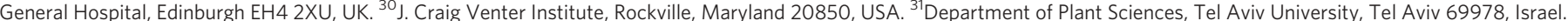

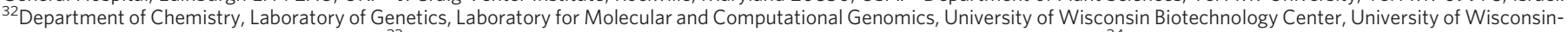

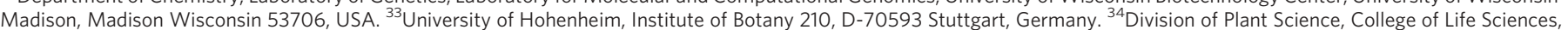

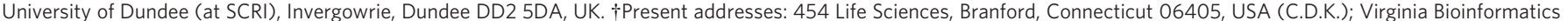
Institute, Virginia Polytechnic and State University, Blacksburg, Virginia 24061, USA (T.T.-A.); Biomedical Diagnostics Institute, Dublin City University, Dublin 9, Ireland (K.O.). *These authors contributed equally to this work.
} 
(95 Mb) and P. ramorum (65 Mb), which cause soybean root rot and sudden oak death, respectively ${ }^{5,6}$. We sequenced the genome of $P$. infestans strain T30-4 using a whole-genome shotgun approach, and generated a ninefold coverage assembly spanning $229 \mathrm{Mb}$ (Table 1 and Supplementary Information). The unassembled fraction of the genome consists of high copy repeat sequences (Supplementary Information). The assembled genome sequence provides near complete coverage of genes, with $98.2 \%$ of $P$. infestans T30-4 complementary DNAs aligning (Supplementary Information). We identified 17,797 protein-coding genes by $a b$ initio gene prediction, protein and expressed sequence tag (EST) homology, and direct genome-togenome comparative gene modelling with $P$. sojae and $P$. ramorum (Supplementary Information). Changes in gene content, number or length do not explain the marked difference in genome size (Table 1 and Supplementary Table 1). No evidence of whole-genome duplication or large-scale dispersed segmental duplication was detected. However, specific disease effector gene families are expanded in $P$. infestans (see later).

P. infestans, P. sojae and P. ramorum represent three major phylogenetic clades of Phytophthora ${ }^{6}$. Among the three genomes, we identified a core set of 8,492 orthologue clusters (including 9,583 $P$. infestans orthologues and close paralogues), of which 7,113 genes show 1:1:1 orthology relationships (Table 1, Supplementary Fig. 1 and Supplementary Table 2). The core proteome is enriched in genes involved in cellular processes including DNA replication, transcription and protein translation, whereas genes with functions involved in cellular defence mechanisms are underrepresented (Supplementary Fig. 2). Differences in gene family expansion, in particular dynamic repertoires of effector genes (see later), are probably responsible for different traits among Phytophthora species, such as altered host specificity.

Comparison of the three Phytophthora genomes reveals an unusual genome organization, comprised of blocks of conserved gene order in which gene density is relatively high and repeat content is relatively low, separated by regions in which gene order is not conserved, gene density is low and repeat content is high (Table 1 and Fig. 1). The conserved blocks represent $\sim 90 \%$ of core orthologous groups in all three genomes, including $\sim 70 \%(12,440)$ of all P. infestans proteincoding genes and $\sim 78 \%$ of genes in both $P$. sojae $(13,225)$ and $P$. ramorum $(11,246)$. Within conserved blocks, genes are typically tightly spaced in all three genomes (Table 1 and Fig. 1), with median intergenic distances of 633 base pairs (bp) for P. ramorum, 804 bp for
$P$. sojae, and $603 \mathrm{bp}$ for $P$. infestans. In regions between conserved blocks, intergenic distances are greater and increase with increasing genome size (median $1.5 \mathrm{~kb}$ for P. ramorum, $2.2 \mathrm{~kb}$ for $P$. sojae, and $3.7 \mathrm{~kb}$ for $P$. infestans). The differences in spacing between genes among the three genomes, within and outside regions of conserved gene order, are evident in Fig. 2a-f. The expansion of regions between conserved blocks results from increased density of repetitive elements (Supplementary Fig. 3), and overall differences in genome size among the three species are largely explained by proliferation of repeats in regions in which gene order is not conserved. This difference between conserved blocks and non-conserved regions is particularly apparent in the greatly expanded $P$. infestans genome (Fig. 2d, f). Further, it is evident that rapidly evolving secreted effector genes (see later) lie predominantly in the gene-sparse regions (Fig. 2g, h). This dual pattern of intergenic spacing and repeat content has been suggested for large, unsequenced genomes in the Poaceae such as maize ${ }^{7-9}$, but it is not seen in the genomes of other sequenced eukaryotes (Supplementary Fig. 4).

Recent proliferation of Gypsy elements in P. infestans underlies the genome expansion. Approximately one-third of the genome assembly corresponds to families of Gypsy elements (Supplementary Fig. 5). The two families with the highest relative expansion in P. infestans are Gypsy Pi-1 and a new Gypsy long terminal repeat (LTR) element we named 'Albatross', which together account for at least 29\% of the genome (Supplementary Table 3 ). Albatross elements cover $\sim 32 \mathrm{Mb}$ and are enriched ( $>2$-fold) in the regions in which gene order is not conserved (Supplementary Table 4 and Supplementary Fig. 6), contributing appreciably to relative expansion of gene-sparse regions (Supplementary Fig. 3). Gypsy Pi-1 elements cover $\sim 22 \mathrm{Mb}$ and, in contrast to Albatross elements, are relatively evenly distributed across the genome.

Overall, the $P$. infestans genome contains a strikingly rich and diverse population of transposons (Supplementary Table 3). We identified 273 full-length elements belonging to two large classes of autonomous rolling-circle type helitron DNA transposons (7.3-kb and 6.4-kb elements), in much larger numbers than described in any other genome (Supplementary Tables 3 and 5). Most helitron open reading frames (ORFs) are degenerate pseudogenes, but 13 are intact and presumed functional. Some apparently non-autonomous helitrons have intact termini so their transposition may be driven by gene products from the functional classes. In contrast, the $P$. sojae and $P$. ramorum genomes contain no intact helitron elements. The $P$. infestans genome carries

Table 1 | Genome assembly and annotation statistics

\begin{tabular}{|c|c|c|c|}
\hline & P. infestans & P. sojae & P. ramorum \\
\hline \multicolumn{4}{|l|}{ Genome } \\
\hline Estimated genome size & $240 \mathrm{Mb}$ & $95 \mathrm{Mb} *$ & $65 \mathrm{Mb}^{*}$ \\
\hline Coverage (fold) & 7.6 & $7.9 *$ & $5.6^{*}$ \\
\hline Number of scaffolds & 4,921 & $1,810 *$ & $2,576 *$ \\
\hline N50 scaffold length & $1,570 \mathrm{~kb}$ & $463 \mathrm{~kb} *$ & 308 kb* \\
\hline Total scaffold length & $228.5 \mathrm{Mb}$ & 86.0 Mb* & $66.7 \mathrm{Mb}^{*}$ \\
\hline Number of contigs & 18,288 & $5,577^{*}$ & $7,588^{*}$ \\
\hline N50 contig length & $44.5 \mathrm{~kb}$ & $105.7 \mathrm{~kb} *$ & $47.5 \mathrm{~kb} *$ \\
\hline Total contig length & $190 \mathrm{Mb}$ & $78 \mathrm{Mb}^{*}$ & $54.4 \mathrm{Mb}^{*}$ \\
\hline $\mathrm{G}+\mathrm{C}$ content & $51.0 \%$ & $54.4 \%$ & $53.9 \%$ \\
\hline Repeat $\uparrow(\%)$ & $74 \%$ & $39 \%$ & $28 \%$ \\
\hline Collinear blockst & $85 \mathrm{Mb}$ & $52 \mathrm{Mb}$ & $37 \mathrm{Mb}$ \\
\hline Repeat $\uparrow(\%)$ in collinear blockst & $57 \%$ & $28 \%$ & $13 \%$ \\
\hline Repeat $\uparrow(\%)$ outside collinear blockst & $86 \%$ & $60 \%$ & $56 \%$ \\
\hline Intergenic region spacing in collinear blocks: (25-75 percentiles) & $224-3,070 \mathrm{bp}$ & $307-2,319 b p$ & $270-1,551 b p$ \\
\hline Intergenic region spacing outside collinear blocksł (25-75 percentiles) & $664-19,144 \mathrm{bp}$ & $753-5,896 b p$ & $566-4,351 \mathrm{bp}$ \\
\hline \multicolumn{4}{|l|}{ Genes } \\
\hline Number of genes§ & 17,797 & 16,988 & 14,451 \\
\hline Phytophthora orthologues & 11,893 & 12,427 & 12,136 \\
\hline Phytophthora core orthologues|| & 9,583 & 9,550 & 9,664 \\
\hline
\end{tabular}

* Statistics derived from supplementary materials accompanying ref. 5.

$\uparrow$ Measured by RepeatMasker with de novo RepeatScout libraries (see Supplementary Information)

t. Union of collinear blocks derived from pairwise genome comparisons (see Supplementary Information).

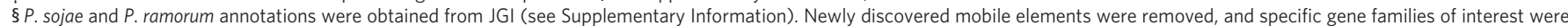
reannotated (see Supplementary Information).

||Core orthologous groups contain at least one orthologous gene from each of the three Phytophthora species. 


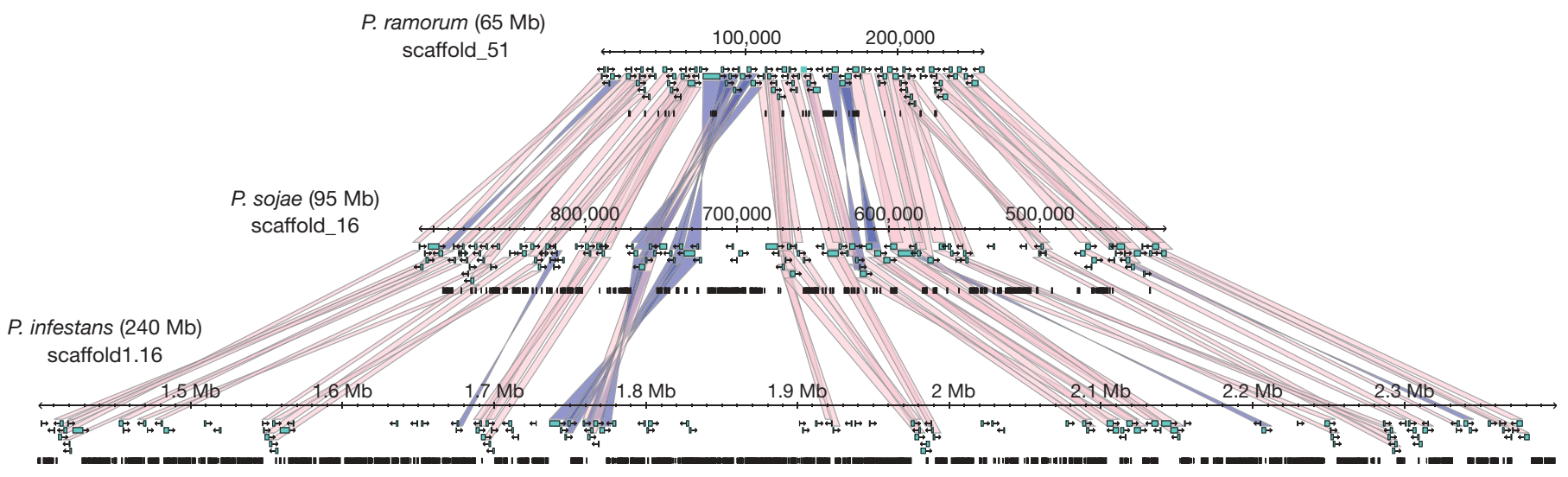

Figure 1 | Repeat-driven genome expansion in Phytophthora infestans. Conserved gene order across three homologous Phytophthora scaffolds. Genome expansion is evident in regions of conserved gene order, a

increased numbers of mobile elements across diverse families as compared to $P$. sojae and $P$. ramorum, with $\sim 5$ times as many LTR retrotransposons and $\sim 10$ times as many helitrons (Supplementary Fig. 7).

Consistent with a model of repeat-driven expansion of the P. infestans genome, the vast majority of repeat elements in the genome are highly similar to their consensus sequences, indicating a high rate of recent transposon activity (Supplementary Fig. 8). In addition, we have observed and experimentally confirmed examples of recently active elements (Supplementary Figs 9-11).

Phytophthora species, like many pathogens, secrete effector proteins that alter host physiology and facilitate colonization. The genome of $P$. infestans revealed large complex families of effector genes encoding secreted proteins that are implicated in pathogenesis ${ }^{10}$. These fall into two broad categories: apoplastic effectors that accumulate in the plant intercellular space (apoplast) and cytoplasmic effectors that are translocated directly into the plant cell by a specialized infection structure called the haustorium ${ }^{11}$. Apoplastic effectors include secreted hydrolytic enzymes such as proteases, lipases and glycosylases that probably degrade plant tissue; enzyme inhibitors to protect against host defence enzymes; and necrotizing toxins such as the Nep1-like proteins (NLPs) and PcF-like small cysteine-rich proteins (SCRs) (Supplementary Table 6).

As in the other Phytophthora species ${ }^{5}$, candidate effector genes are numerous and typically expanded compared to non-pathogenic relatives (Supplementary Table 6). Most notable among these are the RXLR and Crinkler (CRN) cytoplasmic effectors, described later.

The archetypal oomycete cytoplasmic effectors are the secreted and host-translocated RXLR proteins ${ }^{12}$. All oomycete avirulence genes (encoding products recognized by plant hosts and resulting in host immunity) discovered so far encode RXLR effectors, modular secreted proteins containing the amino-terminal motif Arg-X-LeuArg (in which $X$ represents any amino acid) that defines a domain required for delivery inside plant cells ${ }^{11}$, followed by diverse, rapidly evolving carboxy-terminal effector domains ${ }^{13,14}$. Several of these $C$ termini have been shown to exhibit virulence activities as host cell death suppressors ${ }^{15,16}$. We exploited the known motifs and other conserved sequence features to predict 563 RXLR genes in the P. infestans genome (Supplementary Tables 6, 7 and Supplementary Information). RXLR genes are notably expanded in $P$. infestans, with $\sim 60 \%$ more predicted than in $P$. sojae and $P$. ramorum (Supplementary Tables 6 and 7). We observed that 70 of these are rapidly diversifying (Supplementary Table 8). Approximately half of $P$. infestans RXLRs are lineage-specific, largely accounting for the expanded repertoire (Supplementary Figs 12 and 13). In contrast to the core proteome, RXLR genes show evidence of high rates of turnover with only 16 of the 563 genes with 1:1:1 orthology relationships (Supplementary Table 2) and many (88) putative RXLR consequence of repeat expansion in intergenic regions. Genes are shown as turquoise boxes, repeats as black boxes. Collinear orthologous gene pairs are connected by pink (direct) or blue (inverted) bands.

pseudogenes (Supplementary Table 9). This high turnover in Phytophthora is probably driven by arms-race co-evolution with host plants $5,13,14,17$.

RXLR effectors show extensive sequence diversity. Markov clustering (TribeMCL ${ }^{18}$ ) yields one large family ( $P$. infestans: 85 , P. ramorum: 75, P. sojae: 53 ) and 150 smaller families (Supplementary Fig. 14). The largest family shares a repetitive C-terminal domain structure (Supplementary Figs 15 and 16). Most families have distinct sequence homologies (Supplementary Fig. 14) and patterns of shared domains (Supplementary Fig. 17) with greater diversity than expected if all RXLR effectors were monophyletic.

In contrast to the core proteome, RXLR effector genes typically occupy a genomic environment that is gene sparse and repeat-rich (Fig. 2g and Supplementary Figs 18 and 19). The mobile elements contributing to the dynamic nature of these repetitive regions may enable recombination events resulting in the higher rates of gene gain and gene loss observed for these effectors.

CRN cytoplasmic effectors were originally identified from $P$. infestans transcripts encoding putative secreted peptides that elicit necrosis in planta, a characteristic of plant innate immunity ${ }^{19}$. Since their discovery, little had been learned about the CRN effector family. Analysis of the P. infestans genome sequence revealed an enormous family of 196 CRN genes of unexpected complexity and diversity (Supplementary Table 10), that is heavily expanded in P. infestans relative to $P$. sojae (100 CRNs) and P. ramorum (19 CRNs) (Supplementary Table 6). Like RXLRs, CRNs are modular proteins. CRNs are defined by a highly conserved N-terminal 50-amino-acid LFLAK domain (Supplementary Fig. 20) and an adjacent diversified DWL domain (Fig. 3a, b). Most $(60 \%)$ possess a predicted signal peptide. Those lacking predicted signal peptides are typically found in CRN families containing members with secretion signals (Supplementary Table 10). CRN C-terminal regions exhibit a wide variety of domain structures, with 36 conserved domains and a further eight unique $\mathrm{C}$ termini identified among the 315 Phytophthora CRN proteins (Supplementary Table 11). We observed evidence of recombination between different clades as a mechanism driving CRN diversity (Supplementary Figs 21-23).

We explored the ability of diverse CRNs to perturb host cellular processes. In assays for necrosis in planta (Supplementary Information), deletion mutants of the previously described CRN2 secreted protein ${ }^{19}$ defined a C-terminal 234 amino-acid region (positions 173-407, domain DXZ) that is sufficient to induce cell death when expressed inside plant cells (Supplementary Fig. 24). Assays with representative $P$. infestans CRN genes identified four other distinct $\mathrm{C}$ termini that also trigger cell death inside plant cells (Fig. $3 \mathrm{c}$ ). These include the newly defined DC domain ( $P$. infestans: 18 genes and 49 pseudogenes $(\psi))$ and the D2 (14 and $43 \psi)$ and DBF (2 and $1 \psi$ ) domains, which have similarity to protein kinases (Supplementary Table 11). These results indicate that the CRN protein domains 

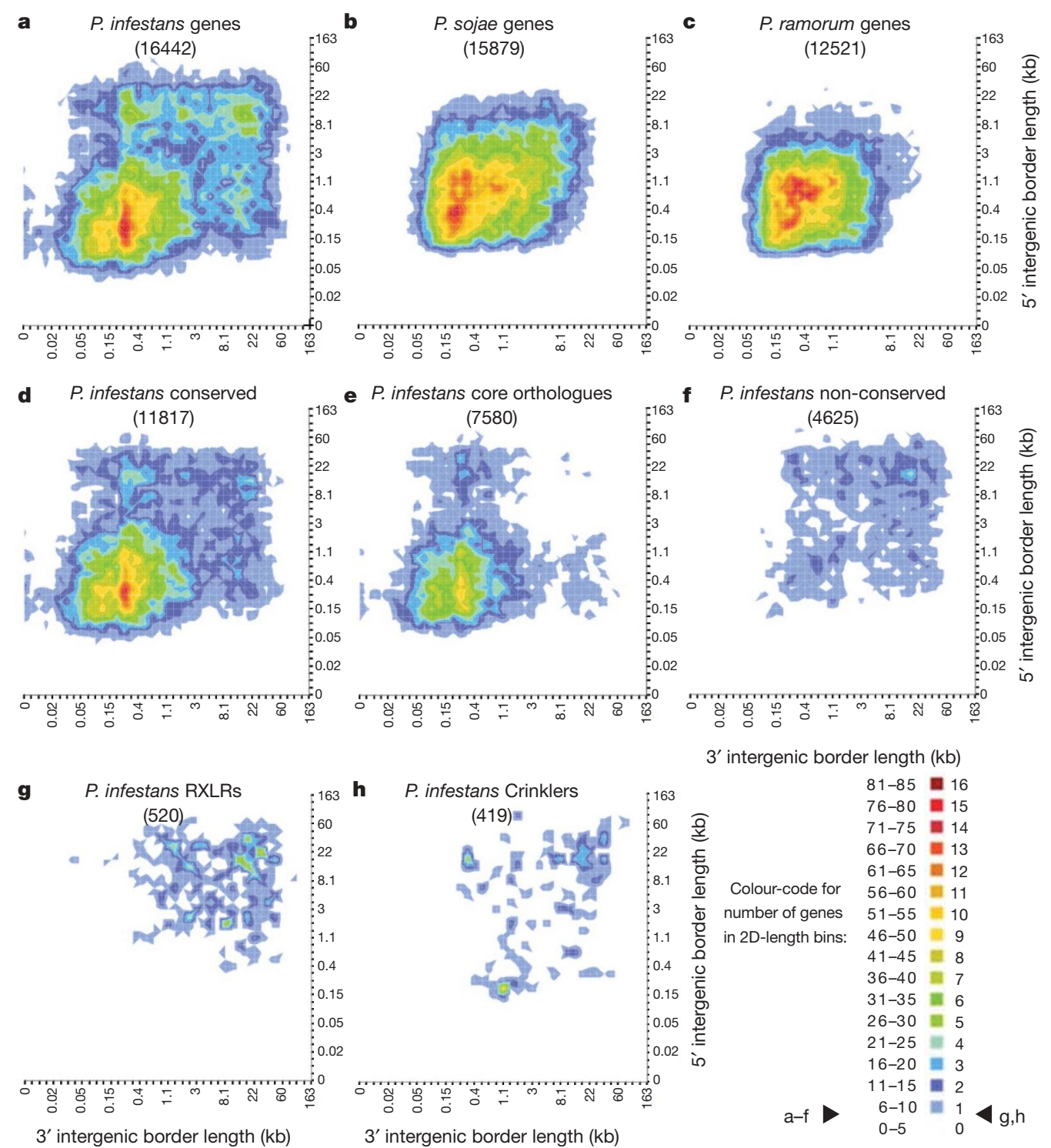

3 ' intergenic border length $(\mathrm{kb})$

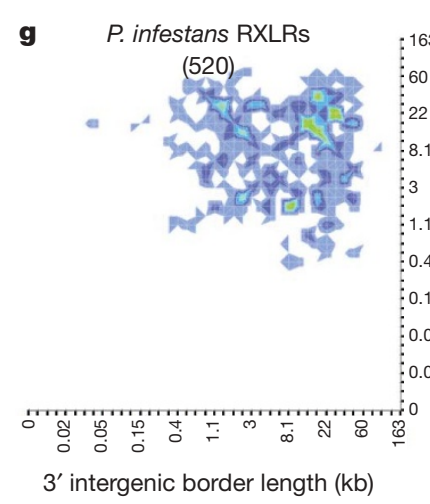

Figure 2 | The $\mathbf{P}$. infestans genome shows an unusual distribution of intergenic region lengths. The flanking distance between neighbouring genes provides a measurement of local gene density. P. infestans genes were sorted into two dimensional bins on the basis of the lengths of flanking intergenic distances to neighbouring genes at their $5^{\prime}$ and $3^{\prime}$ ends. $\mathbf{a}-\mathbf{h}$, The number of genes in each bin is shown as a colour-coded heat map on orthogonal projection. $P$. infestans whole-genome analysis (a) shows most genes with intergenic regions between 20-bp and 3-kb long, as well as sets of genes flanked by one or two intergenic region(s) between $5 \mathrm{~kb}$ and $36 \mathrm{~kb}$.

expressed in planta are retained (lacking signal peptides and hence not secreted) by the plant cell and stimulate cell death by an intracellular mechanism, supporting the view that CRNs, like RXLRs, are cytoplasmic effectors. We propose that the conserved CRN N-terminal LFLAK domain may function similarly to the RXLR motif for delivery of CRN effectors into plant cells, and experiments to test this hypothesis are under way.

A further 255 CRN genes are fragmented or otherwise disrupted and presumably non-functional (Supplementary Table 10). CRN genes and pseudogenes are aggregated in large clusters at several genomic loci, typically clustered by domain type (Supplementary Fig. 25). One extraordinary example is scaffold $1.48(\sim 1.2 \mathrm{Mb})$, containing 21 CRN genes and 31 CRN pseudogenes of the DXZ and D2 necrosis inducing domain-types (Fig. 3d). Many of the pseudogenes show only a few base changes, indicating recent conversion to pseudogenes. This high degree of expansion and pseudogene formation
Comparison with other Phytophthora genomes $(\mathbf{b}, \mathbf{c})$ indicates that this separation is observed in $P$. infestans but not the other two sequenced genomes. Genes in collinear blocks (d) and the core orthologue clusters (e) have primarily shorter intergenic distances, whereas genes outside of collinear blocks (f) reside mostly in gene sparse regions. Genes belonging to the RXLR (g) and Crinkler (CRN) (genes and pseudogenes) (h) effector families have flanking intergenic distances among the longest. Genes found at the ends of scaffolds and hence lacking neighbouring genes were necessarily excluded.

suggests that, like RXLR effector genes, CRN genes have undergone relatively rapid birth and death evolution.

Both CRN and RXLR genes typically occur in repeat-rich, genesparse regions of the genome, where conserved gene order with $P$. sojae and P. ramorum is either absent or disrupted (Fig. $2 \mathrm{~g}, \mathrm{~h}$ and Supplementary Fig. 19). Expansion of large RXLR and CRN effector gene families seems to have been driven by non-allelic homologous recombination and tandem gene duplication. Although the genome is heavily populated by mobile elements, no direct evidence of transposition of effector genes was observed. Instead, the repeat-rich regions of effector clusters probably facilitate non-allelic-homologousrecombination-based expansion. In one intriguing case, nearly identical tandem arrays of CRNs are present on scaffold 1.6 in a perfect head-to-tail arrangement that is similar to that observed for some helitrons (Supplementary Fig. 26). This region of the genome is heavily enriched for helitron elements, implicating helitron-based rolling 
a

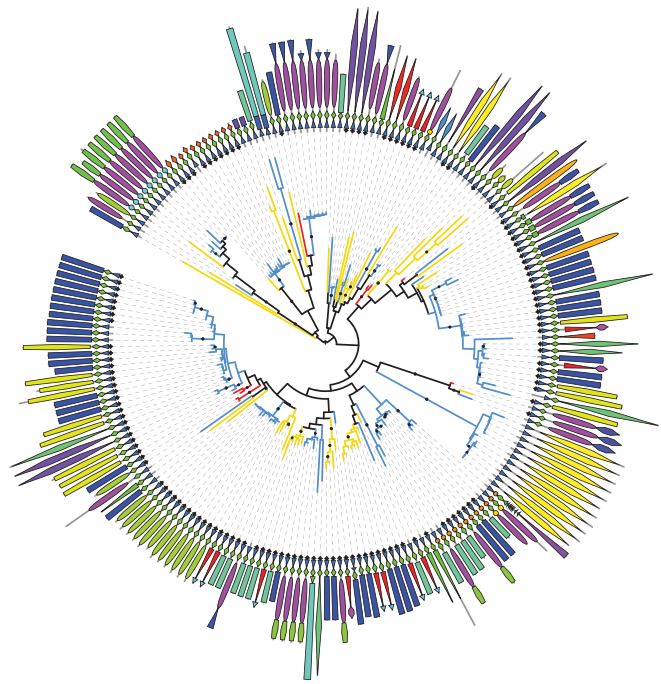

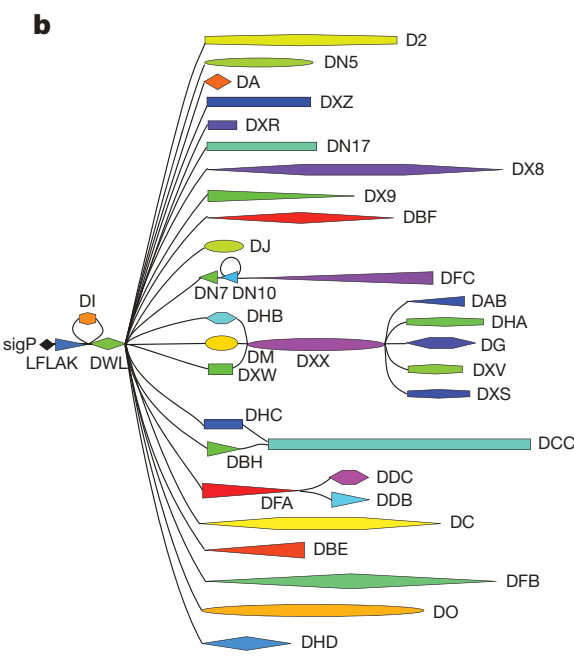

c Control

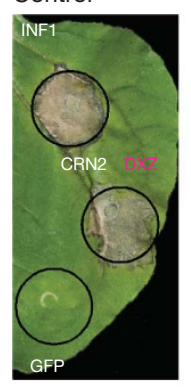

Crinkler effector domains tested
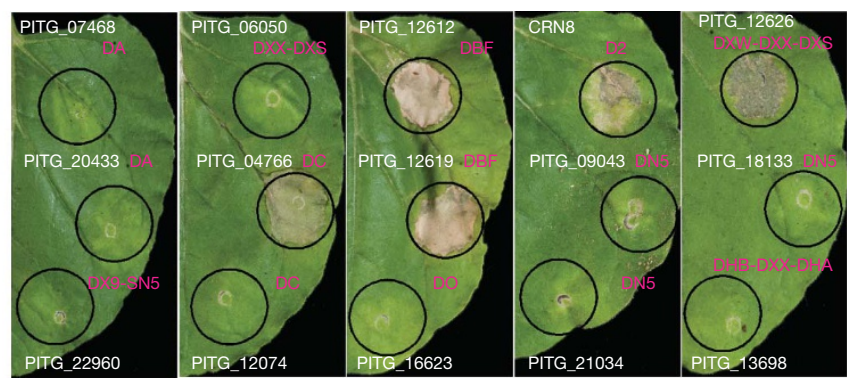

d

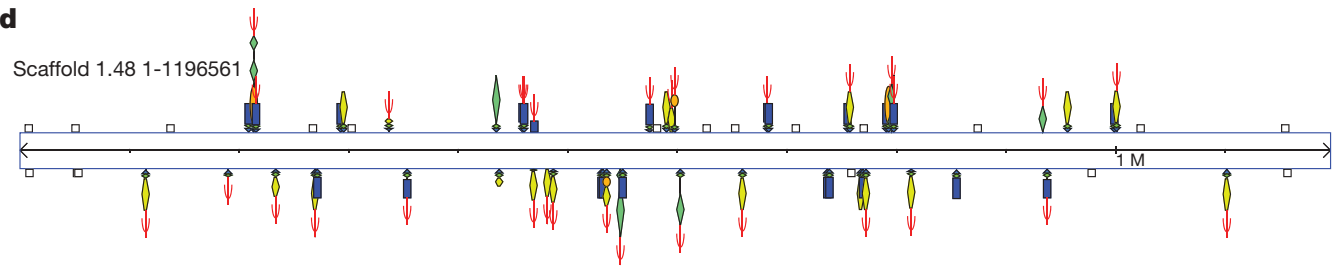

Figure 3 | Diverse Crinkler (CRN) families exhibit necrosis phenotypes in planta. a, CRN family phylogeny on the basis of the conserved N-terminal sequence, computed using PhyML with default parameters and 100 bootstrap replicates. CRN C-terminal domain structures are shown along the circumference. Branches are coloured according to organism: $P$. infestans in blue, $P$. sojae in yellow, and $P$. ramorum in red. Internal nodes with $\geq 80 \%$ bootstrap support are marked with a black dot. $\mathbf{b}$, Graphical representation of the CRN family domain architecture, exhibiting a conserved $\mathrm{N}$-terminal region followed by diverse $\mathrm{C}$-terminal domains. c, Phenotypes observed on Nicotiana benthamiana leaves upon in planta overexpression of CRN effectors. C-terminal effector domains of CRNs were tested for cell death phenotypes on N. benthamiana leaves by Agrobacterium tumefaciens-mediated transient expression of CRNs, infl (positive control), crn2 (positive control), and green fluorescent protein (GFP) (negative control). The domains DC, DBF, D2 and DXW-DXX-DXS, like the DXZ domain of $c r n 2$, were found to induce necrosis. Cell death phenotypes were visible at 4 days post infiltration. Photos were taken 7 days after infiltration. d, CRNs with necrosis domains D2 and DXZ along with pseudogene copies are found co-clustered across $P$. infestans scaffold $1.48(\sim 1.2 \mathrm{Mb})$. Genes and domain structures are illustrated according to the top and bottom strands of the genomic scaffold. Pseudogenes are indicated by $\Psi$; non-CRN genes are shown as unfilled boxes. circle replication as a possible mechanism for establishing this CRN cluster.

To explore transcriptional responses to plant infection, we constructed a NimbleGen microarray based on the genome annotation. $P$. infestans gene expression during potato infection was monitored using samples from infected potato at 2-5 days post-inoculation (d.p.i.). In all, 494 genes were induced at least twofold during infection relative to mycelial growth. Days 2-4 of infection correlate with formation of infectious structures called haustoria. Mycelial necrotrophic growth on dead plant material occurs later at 5 d.p.i., and shows a similar expression profile to mycelial growth in plant extract media (Supplementary Fig. 27a and Supplementary Table 12). Seventy-nine RXLR genes exhibited this pattern of expression, including previously studied avirulence genes Avr3a (ref. 20), Avr4 (ref. 21), and Avr-blb1 (also known as ipiO) (ref. 22) (Supplementary Fig. 27b). Apoplastic effector genes, including protease inhibitors, cysteine-rich secreted proteins, and NPP1-family members, were among the most highly upregulated genes during infection of potato. Few CRNs were induced during infection; however most CRNs were very highly expressed, with $\sim 50 \%$ of CRNs within the top $10 \%$ of gene expression intensities (Supplementary Fig. 28). Several genes encoding metabolic enzymes were upregulated in planta (Supplementary Table 12), suggesting considerable metabolic adaptation of the pathogen to the host environment $^{23}$. A related pattern of downregulation mirrors the induction of effectors, involving $\sim 115$ genes (Supplementary Table 12). Among those repressed were elicitin-like genes and pseudogenes, suggesting that reduced expression during infection or mutation to pseudogene could contribute to evading activation of host innate immunity ${ }^{24}$.

$P$. infestans remains a critical threat to world food security, and the genome sequence is a key tool to understanding its pathogenic success. 
The sequence of the $P$. infestans genome showed an extremely high repeat content $(\sim 74 \%)$ and unusual discontinuous distribution of gene density that correlate intriguingly with its biology. Gene-dense regions with conserved gene order across Phytophthora species are interrupted by repeat-rich expanded regions that are sparsely populated with genes, many of which are fast-evolving pathogenicity effectors such as the RXLR and CRN families. The localization of the effectors to dynamic regions of the genome probably both enables the rapid evolutionary changes and accounts for the considerable expansion in CRN and RXLR effector genes observed in P. infestans. This expansion provides a species-specific repertoire of effector genes, the dynamic nature of which probably provides an advantage in the arms race with host species. We postulate that these dynamic regions promote the evolutionary plasticity of effector genes, generating the enhanced genetic variation required to drive the rapid evasion of plant resistance that is a hallmark of the potato late blight pathogen.

\section{METHODS SUMMARY}

Genomic sequence and gene annotations. The updated $P$. infestans genome sequence and annotation can be accessed through GenBank accession number AATU01000000, and are available through the Broad Institute website at http:// www.broad.mit.edu/annotation/genome/phytophthora_infestans. All genome sequence reads have been deposited in the NCBI trace repository (http:// www.ncbi.nlm.nih.gov/Traces/home/). Paired reads of P. infestans cDNAs are available in dbEST with accessions in the range GR284383-GR301386. The NimbleGen microarray data are available in GEO under accession number GSE14480. Full methods description and associated references are provided as Supplementary Information.

Received 23 April; accepted 31 July 2009.

Published online 9 September 2009.

1. Reader, J. Potato: A History of the Propitious Esculent (Yale Univ. Press, 2009).

2. Haverkort, A. J. et al. Societal costs of late blight in potato and prospects of durable resistance through cisgenic modification. Potato Res. 51, 47-57 (2008)

3. Fry, W. Phytophthora infestans: the plant (and R gene) destroyer. Mol. Plant Pathol. 9, 385-402 (2008).

4. McDonald, B. A. \& Linde, C. Pathogen population genetics, evolutionary potential, and durable resistance. Annu. Rev. Phytopathol. 40, 349-379 (2002).

5. Tyler, B. M. et al. Phytophthora genome sequences uncover evolutionary origins and mechanisms of pathogenesis. Science 313, 1261-1266 (2006).

6. Blair, J. E., Coffey, M. D., Park, S. Y., Geiser, D. M. \& Kang, S. A multi-locus phylogeny for Phytophthora utilizing markers derived from complete genome sequences. Fungal Genet. Biol. 45, 266-277 (2008).

7. Haberer, G. et al. Structure and architecture of the maize genome. Plant Physiol. 139, 1612-1624 (2005).

8. Ma, J. \& Bennetzen, J. L. Rapid recent growth and divergence of rice nuclear genomes. Proc. Natl Acad. Sci. USA 101, 12404-12410 (2004).

9. Yuan, Y., SanMiguel, P. J. \& Bennetzen, J. L. Methylation-spanning linker libraries link gene-rich regions and identify epigenetic boundaries in Zea mays. Genome Res. $12,1345-1349$ (2002)

10. Kamoun, S. A catalogue of the effector secretome of plant pathogenic oomycetes. Annu. Rev. Phytopathol. 44, 41-60 (2006).

11. Whisson, S. C. et al. A translocation signal for delivery of oomycete effector proteins into host plant cells. Nature 450, 115-118 (2007).

12. Morgan, W. \& Kamoun, S. RXLR effectors of plant pathogenic oomycetes. Curr. Opin. Microbiol. 10, 332-338 (2007).
13. Jiang, R. H., Tripathy, S., Govers, F. \& Tyler, B. M. RXLR effector reservoir in two Phytophthora species is dominated by a single rapidly evolving superfamily with more than 700 members. Proc. Natl Acad. Sci. USA 105, 4874-4879 (2008).

14. Win, J. et al. Adaptive evolution has targeted the C-terminal domain of the RXLR effectors of plant pathogenic oomycetes. Plant Cell 19, 2349-2369 (2007).

15. Bos, J. I. et al. The C-terminal half of Phytophthora infestans RXLR effector AVR3a is sufficient to trigger R3a-mediated hypersensitivity and suppress INF1-induced cell death in Nicotiana benthamiana. Plant J. 48, 165-176 (2006).

16. Dou, D. et al. Conserved C-terminal motifs required for avirulence and suppression of cell death by Phytophthora sojae effector Avr1b. Plant Cell 20, 1118-1133 (2008)

17. Qutob, D. et al. Copy number variation and transcriptional polymorphisms of Phytophthora sojae RXLR effector genes Avr7a and Avr3a. PLoS One 4, e5066 (2009).

18. Enright, A. J., Kunin, V.\& Ouzounis, C. A. Protein families and TRIBES in genome sequence space. Nucleic Acids Res. 31, 4632-4638 (2003).

19. Torto, T. A. et al. EST mining and functional expression assays identify extracellular effector proteins from the plant pathogen Phytophthora. Genome Res. 13, 1675-1685 (2003).

20. Armstrong, M. R. et al. An ancestral oomycete locus contains late blight avirulence gene Avr3a, encoding a protein that is recognized in the host cytoplasm. Proc. Natl Acad. Sci. USA 102, 7766-7771 (2005).

21. van Poppel, P. M. et al. The Phytophthora infestans avirulence gene Avr4 encodes an RXLR-dEER effector. Mol. Plant Microbe Interact. 21, 1460-1470 (2008).

22. Vleeshouwers, V. G. et al. Effector genomics accelerates discovery and functional profiling of potato disease resistance and Phytophthora infestans avirulence genes. PLoS One 3, e2875 (2008).

23. Grenville-Briggs, L. J. et al. Elevated amino acid biosynthesis in Phytophthora infestans during appressorium formation and potato infection. Fungal Genet. Biol. 42, 244-256 (2005)

24. Kamoun, S. et al. A gene encoding a protein elicitor of Phytophthora infestans is down-regulated during infection of potato. Mol. Plant Microbe Interact. 10, 13-20 (1997).

Supplementary Information is linked to the online version of the paper at www.nature.com/nature.

Acknowledgements We thank L. Gaffney for help with figures and tables, E. Blanco and R. Guigo for training the GeneID gene prediction software, J. Crabtree for providing a Sybil (http://sybil.sf.net) software component used to render genome comparison illustrations, the Broad Institute Genome Sequencing Platform for sequence data generation, and C. Cuomo and D. Neafsey for comments on the manuscript. The project was supported by the National Research Initiative of the USDA Cooperative State Research, Education and Extension Service, grant numbers 2004-35600-15024 and 2006-35600-16623, and the National Science Foundation grants EF-0333274 and EF-0523670, and the Gatsby Charitable Foundation.

Author Contributions B.J.H., S.K., M.C.Z. and C.N. coordinated genome annotation, data analyses and manuscript preparation. B.J.H. and S.K. made equivalent contributions and should be considered joint first authors (listed by alphabetical order). R.H.Y.J., R.E.H., L.M.C., M.G., C.D.K., S.R., T.T.-A., T.O.B. and K.O. made major contributions to genome sequencing, assembly, analyses and production of complementary data and resources. All other authors are members of the genome sequencing consortium and contributed annotation, analyses or data throughout the project.

Author Information Reprints and permissions information is available at www.nature.com/reprints. This paper is distributed under the terms of the Creative Commons Attribution-Non-Commercial-Share Alike licence, and is freely available to all readers at www.nature.com/nature. Correspondence and requests for materials should be addressed to S.K. (sophien.kamoun@tsl.ac.uk) or C.N. (chad@broad.mit.edu). 